\title{
Aprovechamiento de recursos naturales y culturales con fines turísticos. Caso de estudio: comuna San Jacinto del Pindo, en la provincia de Pastaza (Ecuador)
}

\section{Management of natural and cultural resources for touristic purposes. Study case of San Jacinto del Pindo commune, Pastaza province (Ecuador)}

\author{
María Victoria Reyes Vargas 1,*, Claudia Pamela López León Ging, ," Ángel Fernando Ortega Ocaña, \\ 凶Autor de correspondencia: mreyes@uea.edu.ec, clopezlg@hotmail.com, fortega@uea.edu.ec \\ 1 Universidad Estatal Amazónica, Departamento de Ciencias de la Vida. Puyo-Pastaza-Ecuador. \\ 2 Gobierno Provincial de Pastaza. Puyo-Pastaza-Ecuador. \\ 3 Universidad Estatal Amazónica, Departamento de Ciencias de la Vida. Puyo-Pastaza-Ecuador.
}

Artículo original de la ponencia presentada en el IV CONGRETUR - "El impacto del turismo en el desarrollo del Ecuador", Universidad Central del Ecuador, 21 al 24 de noviembre de 2018

\begin{abstract}
Resumen
El turismo es una actividad de crecimiento sostenido en el mundo, ofrece oportunidades de bienestar económico y desarrollo, así lo confirma la Organización Mundial del Turismo, que refiere un crecimiento del 7\% durante el 2017, superando 1.300 millones de llegadas internacionales, de los cuales arribaron al Ecuador alrededor de 1'600.000 visitantes. El estudio contempló un inventario del potencial turístico de la comuna San Jacinto del Pindo, en la provincia de Pastaza, por medio de visitas de campo, talleres participativos y utilización de fichas de diagnóstico situacional y de caracterización turística, se determinó su situación real, la existencia de planta turística, infraestructura, oferta y la gobernanza en el territorio. Se identificaron los recursos turísticos, conforme lo establece la metodología de inventario del Ministerio de Turismo del Ecuador, lo cual permitió su reconocimiento, registro, clasificación y jerarquización. Este estudio se llevó a cabo en colaboración con el Gobierno Autónomo Descentralizado de la provincia de Pastaza y estudiantes de la carrera de Turismo de la Universidad Estatal Amazónica, como parte de las actividades de servicio comunitario. La ejecución de forma participativa de esta investigación permitió alcanzar resultados que constituyen aportes válidos para la construcción de estrategias de desarrollo y planificación territorial desde la concepción del trabajo colectivo de las comunidades y las instituciones del sector público.
\end{abstract}

Palabras clave: Recurso turístico, Inventario, Comunidad, Diagnóstico.

\begin{abstract}
Tourism is an activity of sustained growth in the world, it offers opportunities for economic welfare and development, as confirmed by the World Tourism Organization, which reports a 7\% growth during 2017, surpassing 1,300 million international arrivals, of which approximately 1'600,000 visitors arrived in Ecuador. The study included an inventory of the tourism potential of the San Jacinto del Pindo commune, in the province of Pasta-
\end{abstract}


za, through field visits, participatory workshops and the use of information sheets on situational diagnosis and tourism characterization. Its real situation was determined by the existence of tourist facilities, infrastructure, offer, and governance in the territory. Tourism resources were identified, as established by the Inventory Methodology of the Tourism Ministry of Ecuador, which allowed for their recognition, registration, classification, and ranking. This study was carried out in collaboration with the Autonomous Decentralized Government of the province of Pastaza and students of the tourism program of the Amazon State University as part of community service activities. The participatory execution of this research allowed to reach results that are valid contributions for the construction of territorial development and planning strategies from the conception of the collective work of the communities and the institutions of the public sector.

Keywords: Tourist resource; Inventory; Community; Diagnosis.

\section{Introducción}

El turismo es una importante actividad que promueve el desarrollo social, económico y político en muchos países, por lo que constituye para las poblaciones una de sus profundas aspiraciones, según lo manifiesta la Organización Mundial del Turismo (OMT, 1999). Es una actividad importante del sector de los servicios, constituye un componente estructural de la sociedad post-industrial, que genera una cantidad importante de empleos y contribuye al producto interno bruto, además de la balanza de pago de muchos países (Martínez, 2010).

Las nuevas motivaciones y tendencias internacionales apuntan hacia una nueva generación en el turismo: el llamado turismo alternativo, del cual son exponentes el turismo basado en la cultura, el patrimonio y la naturaleza, que ofrecen oportunidades para concebir y poner en práctica diferentes modelos viables de desarrollo turístico (Benítez, 2011). Giler (2016), determina que la principal vocación turística de Ecuador está relacionada con la naturaleza y la cultura, es por ello que lo catalogan entre los países megadiversos del planeta por contar con la mayor biodiversidad por unidad de superficie a escala mundial, sus paisajes y riqueza natural lo hacen especialmente atractivo para el turismo.

La región amazónica cuenta con seis provincias, las cuales se caracterizan por su diversidad natural, riqueza cultural y paisajística, cuyos elementos han propiciado el desarrollo y la práctica de varias modalidades turísticas, entre las cuales se destacan las que tienen un enfoque rural, como el turismo de naturaleza, comunitario, gastronómico, de aventura, cultural, entre otras prácticas. Una de las provincias de esta región es Pastaza, la más grande territorialmente hablando, ubicada en el centro de la Amazonía ecuatoriana, la cual se caracteriza por poseer cientos de paisajes y encantos naturales que brindan diferentes opciones a los visitantes. El desarrollo del turismo comunitario es una modalidad que impulsa diversas estrategias que promueven y fortalecen las actividades económicas existentes en este espacio geográfico, permitiendo la diversificación de las labores productivas de sus pobladores, desde una perspectiva participativa y sustentable.

En el afán de profundizar en el conocimiento de la realidad de la actividad turística, en cooperación con la Coordinación de Turismo del Gobierno Autónomo Descentralizado (GAD) de la provincia de Pastaza, se planteó la necesidad de identificar el potencial turístico de varias localidades, entre ellas, las comunidades que conforman la comuna San Jacinto del Pindo, que comprende los cantones Pastaza y Mera; dicha investigación se llevó a cabo con el apoyo de docentes y estudiantes de la carrera de Turismo de la Universidad Estatal Amazónica, así como de técnicos del GAD provincial. El estudio comprende un inventario del potencial turístico, el cual se realizó cumpliendo la metodología del MINTUR (2017) que combina visitas de campo, talleres participativos y utilización de fichas de levantamiento de información validadas previamente por expertos en el tema. La ejecución de forma participativa de esta investigación permitió alcanzar resultados que constituyen aportes válidos para la construcción de estrategias de desarrollo y planificación territorial desde la concepción del trabajo colectivo de las comunidades y las instituciones del sector público.

\section{Materiales y métodos}

La presente investigación es de tipo descriptiva, puesto que recoge información de manera independiente sobre los conceptos o las variables determinadas en el estudio. Se utilizaron los siguientes métodos de investigación:

- Observación: en las visitas de campo realizadas, que permitieron determinar la situación global de la comunidad, en los ámbitos socio-culturales, económicos, ambientales y políticos. 
- Deductivo: su utilización se aplicó en principios descubiertos en casos particulares, a fin de llegar a conclusiones directas sobre el proceso investigativo en la comunidad.

- Descriptivo: en lo que respecta al levantamiento y caracterización de los recursos turísticos inventariados e identificados en la localidad en estudio.

La investigación se basó en el trabajo de campo, que permitió generar la información sobre la situación actual de las comunidades que integran la comuna San Jacinto, la existencia de recursos turísticos naturales y culturales y su aprovechamiento con fines turísticos. El levantamiento de información se realiza en base a la propuesta de diagnóstico de Reyes (2017), la cual resalta la aplicación de encuestas validadas para la recopilación de información en el ámbito social, cultural, ambiental y económico; y la metodología de diagnóstico turístico local de Ricaurte (2009). En lo que respecta al inventario de los recursos turísticos existentes en las comunidades visitadas se utilizó la metodología de Inventario de Atractivos Turísticos (Ministerio de Turismo, 2017), la cual jerarquiza los sitios visitados de acuerdo a los siguientes criterios:

- Criterios relacionados con el índice de competitividad turística:

- $\quad$ Accesibilidad y conectividad

- Planta turística /servicios

- $\quad$ Actividades que se practican (opciones recreacionales)

- Estado de conservación e integración atractivo / entorno

- Políticas y regulaciones

- Salud e higiene

- Seguridad turística

- Criterios relacionados con la demanda:

- $\quad$ Tipo de visitante y afluencia

- Difusión

- $\quad$ Ponderación de criterios

\section{Resultados y discusión}

\subsection{Situación actual de la comuna San Jacinto del Pindo}

La comuna San Jacinto abarca las parroquias de Madre Tierra y Tarqui, ubicadas en los cantones de Mera y Pastaza, en la provincia de Pastaza, región amazónica. La comuna se encuentra en la parte alta de la cuenca hidrográfica del río Pastaza, presenta una temperatura media anual que varía entre $17,9^{\circ}$ a $22,6{ }^{\circ} \mathrm{C}$. y una pluviosidad entre los $1.251 \mathrm{~mm}$ a $4.746 \mathrm{~mm}$, corresponde a la formación vegetal bosque siempre verde montano bajo. La población de la comuna corresponde en un $93 \%$ a la nacionalidad Kichwa, 6\% Shuar y 1\% Achuar (Lamiña, 2014).

La base social del pueblo Kichwa de Pastaza son las familias, conocidas como ayllukuna, que están integradas por familias ampliadas que comparten lazos de consanguinidad y afinidad, siendo la cabeza de familia generalmente un anciano. La unión de diversas familias forma una comunidad, y la unidad entre comunidades está determinada por las familias que la entrelazan generando una participación activa de las comunidades en la defensa de sus territorios y la búsqueda de nuevos procesos de desarrollo social, ambiental, económico, político y cultural (Vitery, 2010).

San Jacinto del Pindo es una organización de segundo grado, una de las más antiguas y grandes de la provincia de Pastaza, su fundación se registra en el año 1944, durante la administración de Velasco Ibarra (Silva, 2003). La adjudicación de sus territorios se da el 12 de marzo de 1947 bajo la gestión de misioneros dominicos y del curaca Severo Vargas, bajo la Ley de Comunas, quienes logran legalizar 40.000 hectáreas para esta comuna.

La comuna está conformada por 37 comunidades (Figura 1), de las cuales 15 fueron sistematizadas, puesto que en las mismas se evidenció la existencia de recursos naturales y culturales con posibilidad de ser aprovechadas en el ámbito turístico. Las comunidades estudiadas son las siguientes: Yana Amarun, Chorreras, Campo Alegre, Ilupungo, Mushuk Warmi, Amazonas, Nueva Vida-Mushu Kawsay, Puerto Santa Ana, Putuimi, Chinimbi, La Encañada-Chuva Urku, San Pedro (Lisan Wasi), San Jacinto, Dos Ríos y Playas del Pastaza.

El desenvolvimiento social y económico de las comunidades cercanas a la ciudad está condicionado a las actividades de mercado y comercio de la zona urbana, mismas que han modificado sus prácticas ancestrales de uso y manejo de los recursos del bosque, debido a los modelos económicos vigentes que promueven la generación de ingresos económicos basados en la creación de monocultivos, pastizales y ganadería, explotación forestal, entre otras actividades (Vitery, 2010). El eje socio económico de las comunidades de San Jacinto se basa principalmente en la producción agrícola, la ganadería, la piscicultura y extracción selectiva de madera. 
La dinámica de los asentamientos comunitarios presenta diferencias en razón del crecimiento demográfico de estas comunidades y la falta de planificación en la construcción de infraestructuras de servicios comunitarios; ocasionando impactos en el entorno ambiental, la economía, la cultura y la organización social. Por tanto, los sistemas de asentamiento y de vida comunitario ancestral están cambiando; y en la actualidad no existen procesos de planificación y gestión sostenible de territorios y recursos naturales en todo el territorio de la comuna San Jacinto.

Entre los principales impactos que se identifican en los últimos años se destacan los problemas generados por apertura de vías, aumento de comunidades, crecimiento poblacional, sobreexplotación de material pétreo, expansión de la frontera agrícola y ganadera, descargas contaminantes en los ríos, entre otros. Los pobladores afirman que hace una década atrás la mayoría de sus habitantes se dedicaban a la venta de madera (por hectáreas), es decir, el valor variaba de acuerdo al volumen y calidad de la madera, siendo superficies considerables las que se comercializaban (entre 1 y 40 hectáreas); entre los principales mercados de compra se hace referencia a los provenientes de las ciudades de Puyo, Ambato, Riobamba y Cuenca.

En la actualidad, los pobladores afirman vivir en una situación precaria e insostenible, es evidente la escasez de recursos de flora y fauna en la zona, puesto que indican que las actividades de explotación y venta de madera persiste hasta la actualidad, que si bien no es en la misma magnitud de épocas remotas, aún constituye un problema importante en el territorio. Se hace referencia que solo una comunidad (Campo Alegre) es la que ha emprendido en el programa de reforestación del Gobierno nacional con las especies de laurel, cedro, ahuano, canelo, entre otros, dentro del Programa estatal Socio Bosque.

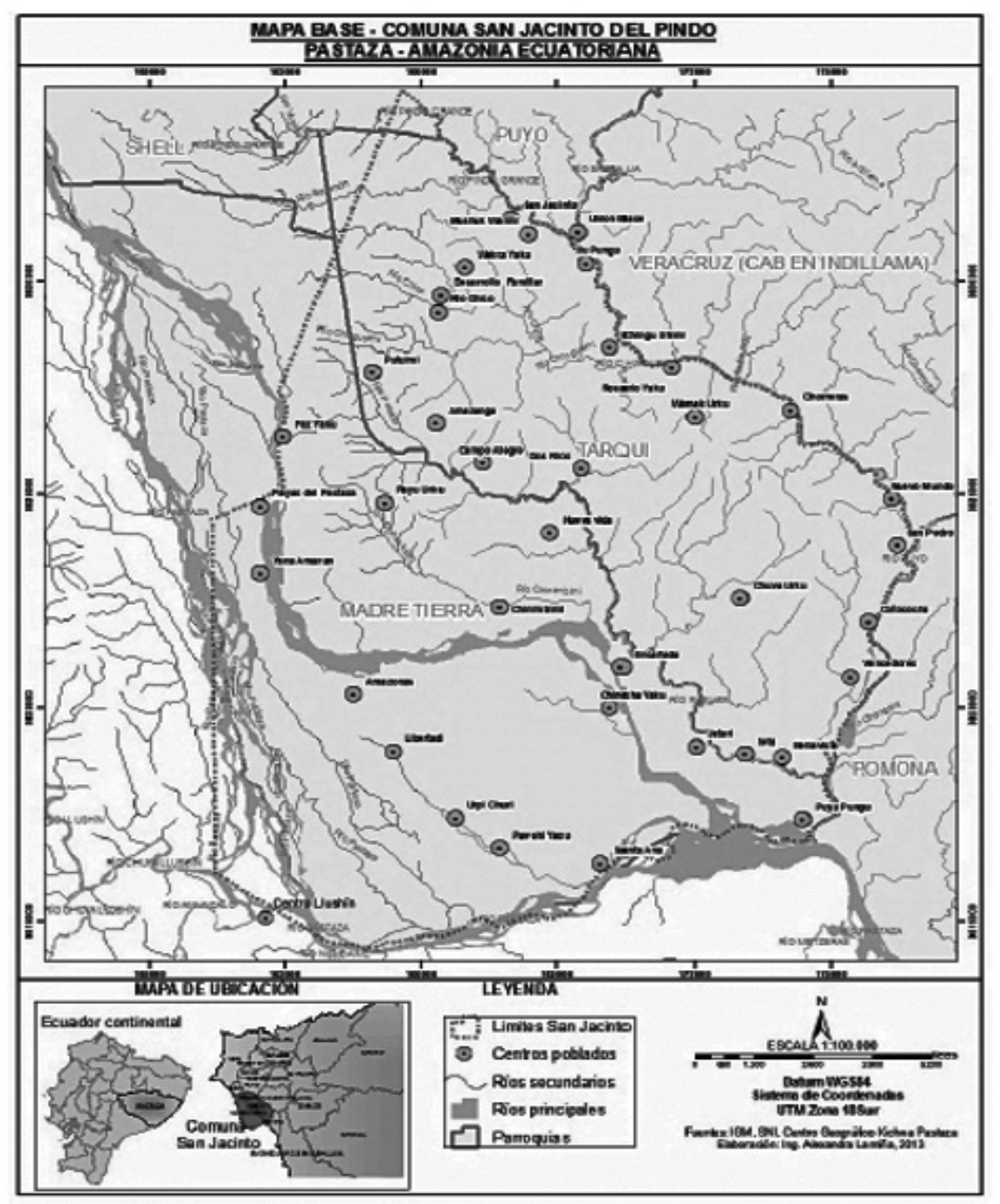

Figura 1. Comunidades que conforman la comuna San Jacinto del Pindo (Lamiña, 2014). 
En lo que se refiere a recuperación y conservación de recursos de fauna, son pocas las iniciativas que han emprendido las comunidades, más bien los emprendimientos comunitarios se orientan al turismo, ya que dicha actividad de alguna forma ha contribuido a concienciar a la población sobre la importancia de recuperar y conservar los bosques y por ende sus recursos, ya que constituyen la base de la oferta turística en lo que se refiere al aprovechamiento artesanal, gastronómico, actividades culturales, expresiones y manifestaciones de la cultura, que son parte de los ingresos económicos actuales de varias de las comunidades.
En lo referente a la forma de economía comunitaria existente en la localidad, se identificaron dos ámbitos: la economía de subsistencia Kichwa y la economía rural comunal o trabajo colectivo. La primera se caracteriza por presentar elementos referentes a la chacra, sitios de pesca, rutas de cacería y áreas para la extracción de materiales e insumos para la artesanía; mientras que la economía rural está representada por programas y proyectos que ejecutan las comunidades propiamente (figuras 2 y 3$)$.

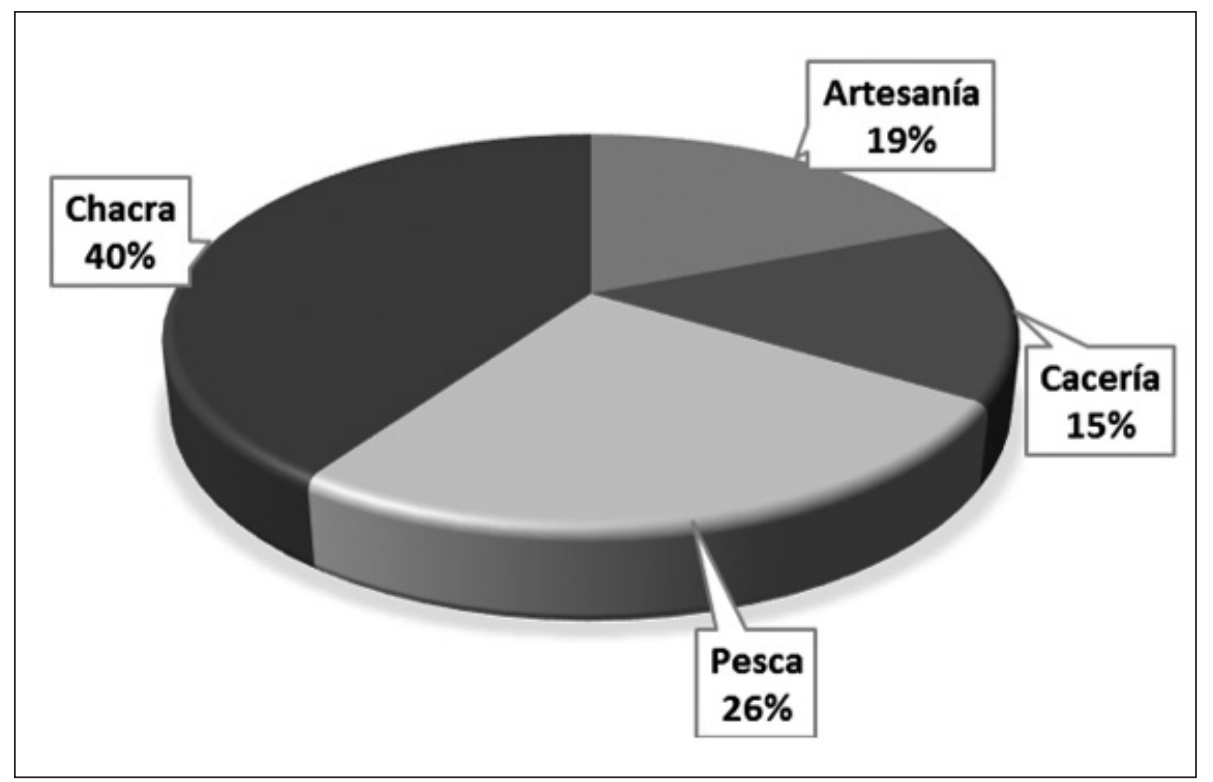

Figura 2. Actividades económicas tradicionales Kichwa.

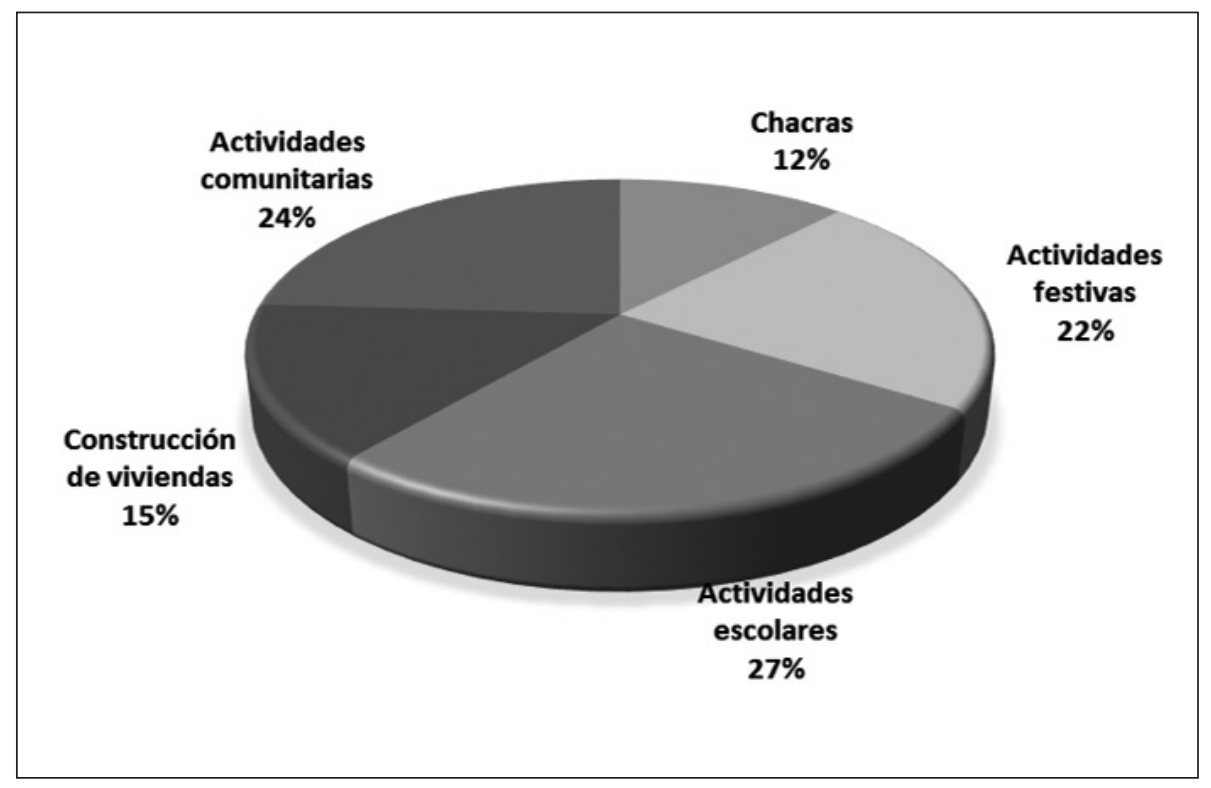

Figura 3. Trabajo colectivo. 
En lo referente a la economía de subsistencia se aplicó una encuesta con respuestas múltiples. La figura 2 muestra que el $40 \%$ de la población encuestada posee, al menos, una chacra; el 26\% manifiesta continuar practicando la pesca tradicional; el $15 \%$ de la población practica la cacería de especies silvestres como guantas, guatusas, armadillos, etc. En lo referente a la artesanía, el 19\% de los encuestados aseveran que se trata de una actividad medianamente rentable, manifiestan que requieren de capacitación y tecnificación de las piezas artesanales que producen. En la figura 3, que se refiere al trabajo colectivo (conocido como minga), se resalta que la población se dedica a colaboración en actividades festivas (22\%), mejoramiento de la infraestructura de escuelas en actividades escolares (27\%), actividades comunitarias, como limpieza de caminos, infraestructura comunitaria y canchas deportivas (24\%), construcción de viviendas (15\%), apertura de chacras (12\%), esta última de ayuda familiar más no comunitario.

La población encuestada, a través de respuestas múltiples, indicó que las principales actividades económicas (Figura 4) a las que se dedican son la participación en proyectos agroforestales, ya sea con manejo de cultivos de yuca, maíz, plátano, papachina y en menor cantidad especies medicinales y forestales y frutas tropicales, sin dejar de lado los monocultivos de caña, naranjilla y pasto para la ganadería. En el ámbito artesanal, la población encuestada indicó que se dedican a elaborar productos con semillas, bejucos, arcilla, plumas de aves, mullos, tallados de madera de balsa, diseño de instrumentos musicales tradicionales, artefactos de cacería, entre otros elementos disponibles en el territorio; siendo la comercialización de los mismos una tarea básicamente de las mujeres de las comunidades

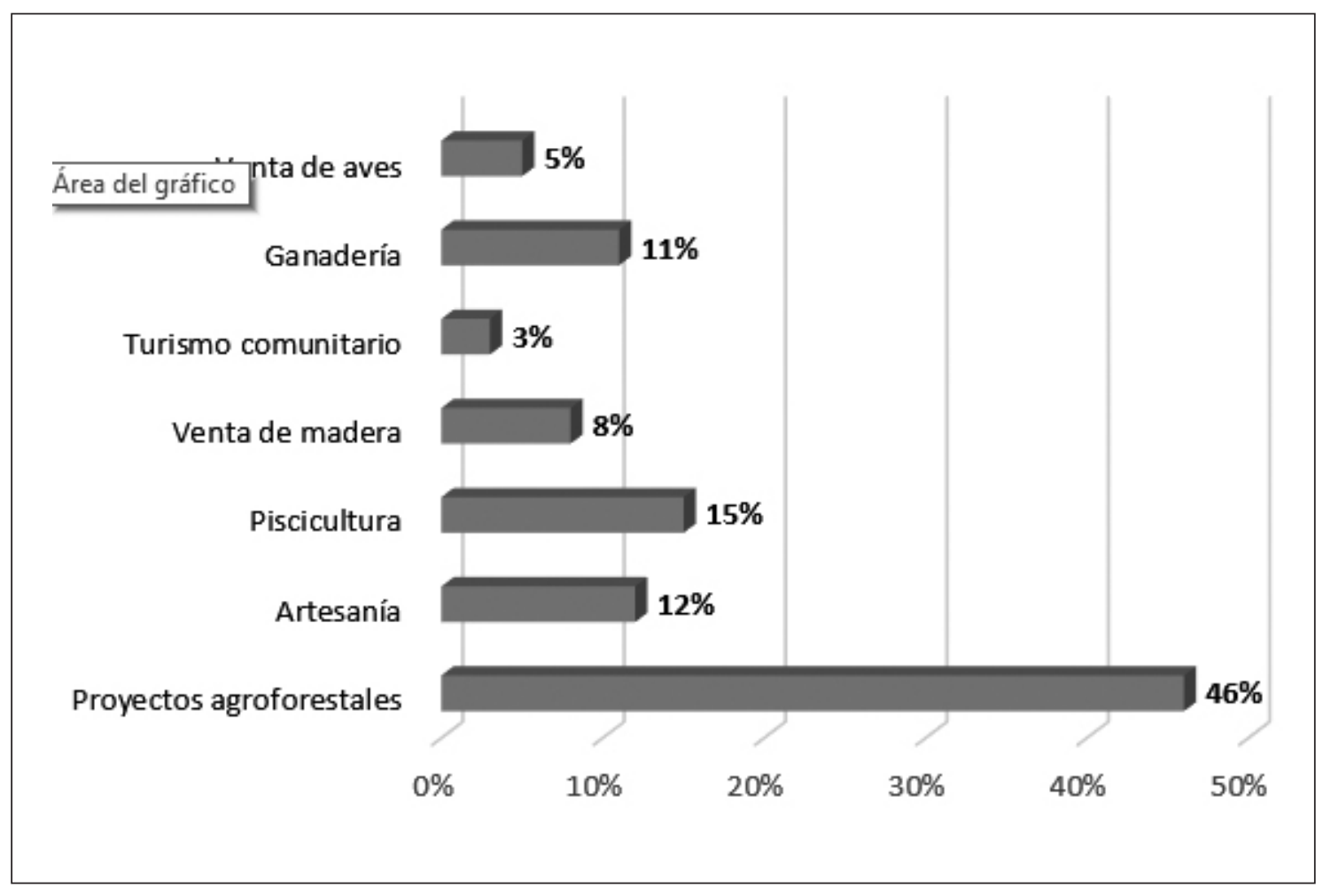

Figura 4. Principales actividades económicas.

En lo referente a la piscicultura, la población afirmó que es una actividad relativamente nueva en las comunidades, puesto que la mayoría cultiva la tilapia con fines de consumo familiar. La madera que se comercializa como parte de la dinamización económica de las comunidades, es el guarumo, balsa, intachi, etc., estas especies se explotan en bosques secundarios que dispo- ne la comuna, regularmente la venta de dicha madera no responde a un plan de manejo, puesto que las familias las ofertan conforme a sus necesidades habituales.

En lo referente al turismo, la población encuestada determina que en la actualidad genera beneficios económicos de forma mediana, puesto que la gran parte de sitios de visita únicamente son recursos turís- 
ticos y no cuentan con servicios, ni facilidades turísticas que complementen las necesidades de visitación.

\subsection{Recursos turísticos identificados en la comuna San Jacinto del Pindo}

Conforme lo determina la metodología de inventario turístico (MINTUR, 2017) es un registro valorado de los sitios que, por sus atributos naturales, culturales y oportunidades para la operación constituyen parte del patrimonio nacional; dicho instrumento permitió la identificación, clasificación y valoración de los escenarios con mejores condiciones para el desarrollo de futuros productos turísticos.

Las visitas de campo realizadas a 15 comunidades sistematizadas, permitió identificar 31 sitios turísticos (tablas 1 y 2), que conforme la clasificación de atractivos turísticos se ubican 21 en la categoría de sitios naturales y 10 sitios en manifestaciones culturales, cada una con su tipo y subtipo (Figura 5), siendo la comunidad La Encañada la que mayor riqueza natural posee, y las comunidades de Chinimbi y Yana Amarun las que mayor presencia de manifestaciones culturales ofrecen al visitante.

En la categoría de sitios naturales se reconocen las siguientes tipologías: montañas, planicies, desiertos, ambientes lacustres, ríos, bosques, aguas subterráneas, fenómenos espeleológicos, fenómenos geológicos, costas o litorales, ambientes marinos y tierras insulares; en la categoría manifestaciones culturales se reconocen las tipologías: arquitectura, folklore, realizaciones técnicas y científicas y acontecimientos programados.

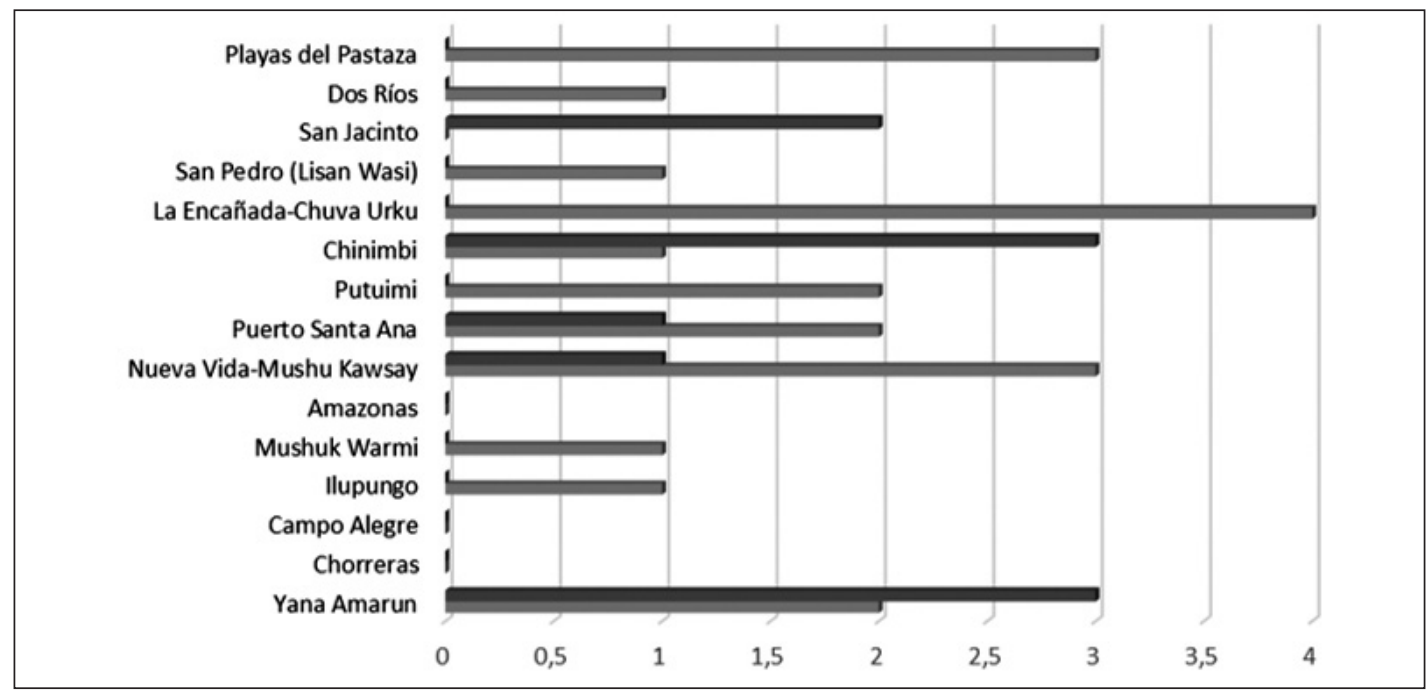

Figura 5. Categoría de sitios turísticos identificados.

Tabla 1. Sitios naturales de interés turístico identificados

\begin{tabular}{|c|c|c|c|}
\hline Comunidad & Sitios naturales & Tipo & Subtipo \\
\hline \multirow{2}{*}{ Yana Amarun } & Río Yana Amarun & Río & Río \\
\hline & Laguna Yana Amarun & Ambiente lacustre & Laguna \\
\hline Chorreras & \multicolumn{3}{|c|}{ No facilita } \\
\hline Campo Alegre & \multicolumn{3}{|c|}{ No facilita } \\
\hline Ilupungo & Cascada Cari Pakcha & Caída de agua & Cascada \\
\hline Mushuk Warmi & Río Pindo & Río & Ribera \\
\hline Amazonas & \multicolumn{3}{|c|}{ No facilita } \\
\hline \multirow{3}{*}{ Nueva Vida-Mushu Kawsay } & Sendero natural & Sendero & Sendero \\
\hline & Dique Nueva Vida & Río & Vado \\
\hline & Cascada & Río & Cascada \\
\hline \multirow{2}{*}{ Puerto Santa Ana } & Bosque protector Yawa Jee & Parques Nacionales & Área protegida \\
\hline & Río Pastaza & Río & Río \\
\hline
\end{tabular}




\begin{tabular}{lccc}
\hline \multirow{2}{*}{ Putuimi } & Río Pituimi & Río & Río \\
& Laguna Caimán & Ambiente lacustre & Laguna \\
& Río Chinimbi & Río & Río \\
\hline \multirow{3}{*}{ La Encañada-Chuva Urku } & Cascada & Río & Cascada \\
& Cueva de murciélago & Fenómeno espeleológico & Cueva \\
& Río Chuva & Río & Río rápido \\
& Montaña Chuva Urku & Montaña & Baja montaña \\
\hline San Pedro (Lisan Wasi) & Cascada de la comunidad & Ríos & Cascada \\
\hline Dos Ríos & Río Chingushimi & Río & Riachuelo \\
\hline \multirow{2}{*}{ Playas del Pastaza } & Cascada Supay Ricuna & Río & Cascada \\
& Mirador Shiwacocha & Media montaña & Mirador \\
& Mirador Juri Juri & Media montaña & Mirador \\
\hline
\end{tabular}

Tabla 2. Manifestaciones culturales de interés turístico identificados

Yana Amarun

Comunidad

Sitios Culturales

Artesanías

Fiesta de aniversario

Chacras nativas

\begin{tabular}{lccc}
\hline Chorreras & No facilita & \\
\hline Campo Alegre & & No facilita & \\
\hline Amazonas & No facilita & \\
\hline Nueva Vida-Mushu Kawsay & Artesanías & Folklore & Artesanías y artes \\
\hline Puerto Santa Ana & Danza tradicional & Música y danza & Cerámica \\
\hline & Elaboración de cerámica & Folklore & Fiesta religiosa \\
Chinimbi & Fiesta del Niño Jesús & Folklore & Medicina ancestral \\
& Medicina ancestral & Folklore & Explotaciones industriales \\
\hline San Jacinto & Panelera San Jacinto & Realizaciones técnicas & Arquitectura civil
\end{tabular}

\section{Subtipo}

Artesanías y artes

Fiestas religiosas

Folklore

Explotaciones agropecuarias

. 


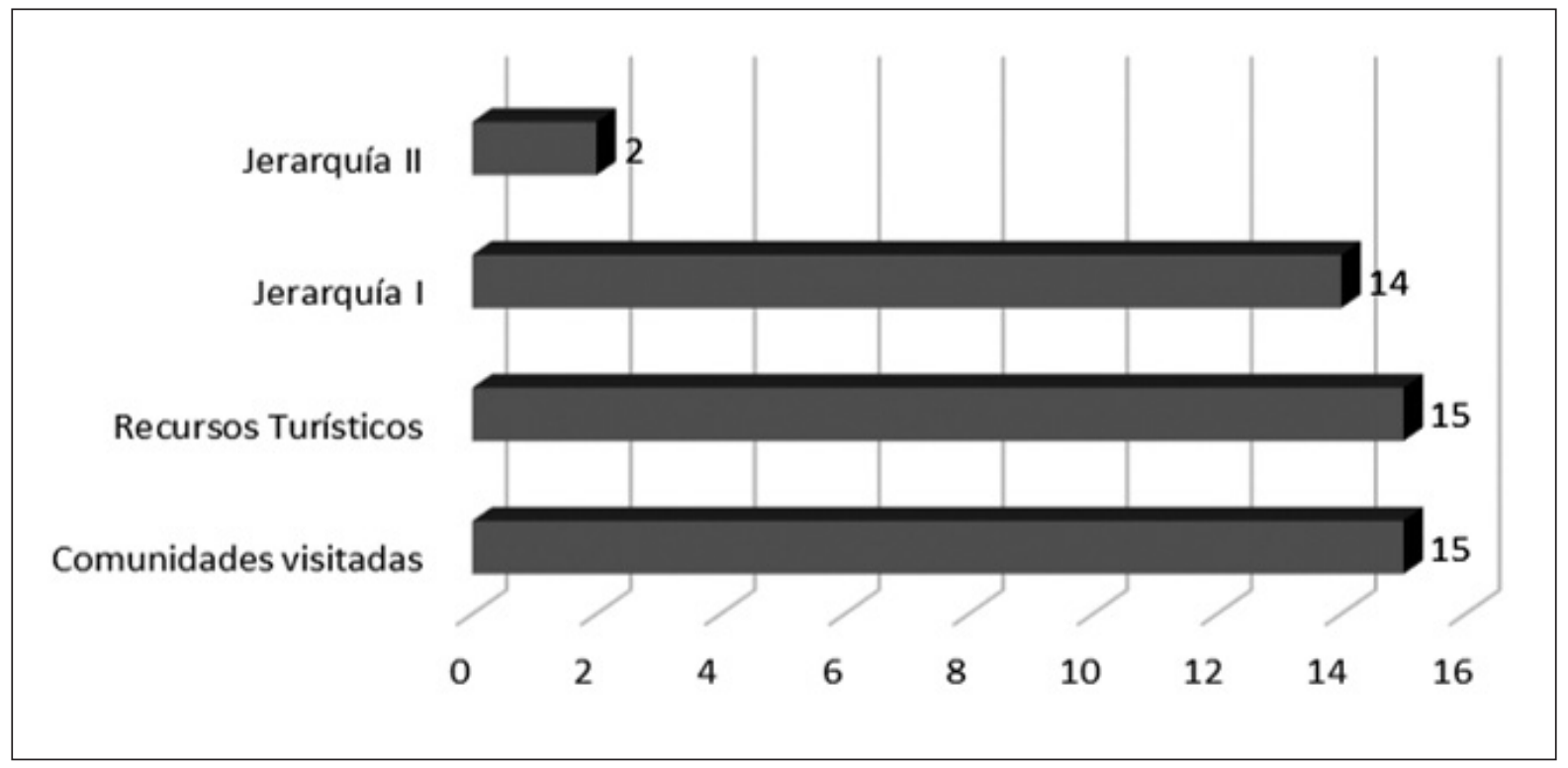

Figura 6. Jerarquización de sitios turísticos identificados.

Finalmente, es evidente que el manejo de los recursos naturales que los grupos indígenas conciben dentro de sus territorios, delimitados por posesión ancestral, les permite garantizar la permanencia de su población y asegurar su sobrevivencia basada en formas de vida tradicionales de relación directa con la naturaleza. Sin embargo, en la actualidad, en lo que respecta al contexto de la globalización de la economía, esta relación se ha mercantilizado dando paso a la exclusión de los pueblos indígenas y de sus prácticas tradicionales de aprovechamiento de los recursos naturales, así como a procesos de degradación de la naturaleza. Motivaciones que han propiciado las demandas de los pueblos en contra de esta mercantilización y discriminación, la cual se basa en la autonomía y la autodeterminación (Criollo, 2017), con el fin de, lograr reapropiarse de su patrimonio natural y cultural y desarrollar procesos productivos orientados a eliminar la pobreza y a alcanzar niveles de autosuficiencia a través de la autogestión de sus recursos, siendo el turismo una de dichas alternativas.

Es evidente que las condiciones de existencia de las comunidades dependen de la legitimación de los derechos de propiedad de las poblaciones sobre su patrimonio de recursos naturales y de su propia cultura y por la redefinición de sus procesos de producción, sus estilos de vida y sus sentidos existenciales conforme lo afirma Leff (2004), sin embargo, la autonomía de los pueblos indígenas establece "Un régimen político-jurídico, acordado y no meramente concedido, que implica la creación de una verdadera colectividad política en el seno de la sociedad nacional" (Díaz, 1993). Es decir que se configura un gobierno propio o autogobierno con absoluta independencia, con capacidades y atribuciones legalmente establecidas para legislar su vida interna y administrar sus recursos en un territorio determinado. Sin embargo y dependiendo de las características de las comunidades, los sistemas autonómicos son diferentes y deben ser evaluados, para su aplicación, en términos de las condiciones históricas de donde emergieron y que, al mismo tiempo quieren dar respuesta, justamente a través de la construcción o reconstrucción de sus formas de vida, es decir, a partir de lo cotidiano.

En las visitas de campo se evidenció la degradación ambiental, la marginación social y la pobreza existente en este espacio territorial, lo cual se pone de manifiesto con la crisis ambiental mundial generada por la racionalidad económica fundada en la lógica del mercado capitalista, con el subsecuente control de los recursos naturales a través de los aparatos de control económico y político-ideológico del Estado o apadrinados por él.

En general, el movimiento indígena, dentro de este panorama, se posiciona en la panacea de los movimientos ambientalistas que, a través de la articulación decisiva con las propuestas de este y otros sectores, que han sido directamente afectadas en sus estilos de vida (pérdida de sus prácticas culturales, emigración territorial, marginación social, explotación económica desempleo, inaccesibilidad a los servicios públicos, destrucción de sus recursos naturales, pérdida de sus medios de subsistencia) han encontrado nuevas formas de organización social para afrontar la crisis ambiental, tomando en cuenta que 
la satisfacción de las necesidades materiales y espirituales dependen en mayor grado de sus condiciones ecológicas y culturales locales de sustentabilidad.

De aquí que, las propuestas de autonomía indígena, desde sus identidades y culturas forman parte indispensable de un proceso local para la implementación de estrategias que respondan a una perspectiva de la racionalidad ambiental que toma como base las condiciones de diversidad ecológica y cultural, permitiendo un aprovechamiento racional de los recursos existentes, con fines turísticos, como una actividad complementaria a las que habitualmente se dedica la población.

\section{Conclusiones}

Se sistematizaron un total de 15 comunidades de las 37 que pertenecen a la comuna San Jacinto del Pindo: Yana Amarun, Chorreras, Campo Alegre, Ilupungo, Mushuk Warmi, Amazonas, Nueva Vida-Mushu Kawsay, Puerto Santa Ana, Putuimi, Chinimbi, La Encañada-Chuva Urku, San Pedro (Lisan Wasi), San Jacinto, Dos Ríos y Playas del Pastaza. En los sitios visitados se realizó la caracterización de la comunidad, en donde se recopiló información referente a población, servicios de educación y salud, transporte, acceso, gobernanza, necesidades de cada comunidad y potencialidades turísticas en el contexto natural y cultural.

En las comunidades visitadas se identificó un total de 31 atractivos turísticos, de los cuales 21 corresponden a sitios naturales y 10 son manifestaciones culturales. Luego de ser jerarquizado conforme la metodología de inventario del MINTUR (2017) cada uno de los recursos, se obtuvo que 15 de los sitios visitados son considerados recursos turísticos, es decir, cuentan con elementos naturales o cultu-

\section{Referencias}

Benítez, J.M. (2011). Tendencias del turismo. Conferencia dictada en la Universidad Central de las Villas, 30 de noviembre de 2011.

Criollo, M. (2007). Manejo de recursos naturales de autonomía indígena amazónica, caso OPIP y el plan de vida en la comunidad de San Jacinto del Pindo-Provincia de Pastaza. Facultad Latinoamericana de Ciencias Sociales FLACSO.

Díaz, P. (1993). Autonomía regional, la autodeterminación de los pueblos indios. México: Siglo Veintiuno editores. rales que pueden motivar el desplazamiento, pero aún no se encuentran incorporados en la dinámica turística, ni cuentan con ningún tipo de infraestructura de apoyo; 14 sitios visitados se encuentran en Jerarquía I, es decir, son atractivos sin mérito suficiente para motivar la visita; y únicamente 2 sitios corresponden a Jerarquía II, lo cual significa que son atractivos con algún rasgo llamativo, capaz de interesar a visitantes que hubiesen llegado a la zona por otras motivaciones turísticas, o de motivar corrientes turísticas nacionales.

De los 31 sitios visitados, únicamente 11 cuentan con ciertos servicios de alojamiento, alimentación, guianza y muy reducidos casos de operación y transporte turístico.

Es evidente, las necesidades identificadas en el territorio, las cuales responden a los requerimientos de mejoramiento de los servicios turísticos, que se enfocan en el mejoramiento o construcción de nueva infraestructura en los sitios que brindan cierto tipo de servicios, ya que en su gran mayoría no tienen aprovechamiento turístico. En lo que se refiere a la administración y comercialización, se enfocan en la planificación; en lo referente a servicios básicos, es palpable la carencia que existe de servicios básicos, primero como satisfacción humana y luego para brindar un óptimo servicio turístico a quienes visitan la zona.

La gran parte de las comunidades visitadas no cuentan con mayor potencial turístico, razón que ha propiciado el que esta actividad no sea considerada como importante dentro de las actividades económicas de estas poblaciones; sin embargo, en varias de ellas, sobre todo en las que de alguna forma tienen una ponderación significativa, se requiere analizar la posibilidad de intervención para promover una nueva fuente de alternativa económica para el desarrollo de estas poblaciones.

Giler, D. (2016). Turismo PRO ECUADOR. Obtenido de: http://www.proecuador.gob.ec/ sector $12 /$

Lamiña, M. (2014). Estudio de diagnóstico territorial y de asentamientos humanos de la comunidad Kichwa San Jacinto del Pindo, proyecto de construcción de la CTI Nacionalidad Kichwa de Pastaza. Puyo-Ecuador.

Leff, E. (2004). Racionalidad ambiental, la reapropiación social de la naturaleza. México: Siglo XXI. 
Martínez, M. (2010). Las nuevas tendencias del turismo. El Salvador: Universidad Francisco Gavidia, Facultad de Ciencias Económicas.

MINTUR, (2017). Manual para la realización del inventario de atractivos y espacios turísticos del Ecuador. Quito-Ecuador: Ministerio de Turismo.

Organización Mundial del Turismo. (1999). Introducción al turismo. Madrid-España, Organización Mundial del Turismo.

Reyes, M. (2017). Propuesta metodológica para la evaluación de recursos turísticos rurales de la
Amazonía ecuatoriana, caso Pastaza. Ponencia en el Décimo Primer Congreso Internacional de Turismo ESPOCH, Junio 27, 28, 29, 30 del 2017. Riobamba-Ecuador

Ricaurte, C. (2009). Guía Manual del diagnóstico turístico local. Guayaquil-Ecuador: Escuela Superior Politécnica del Litoral.

Silva, E. (2003). Mushuc Allpa. Quito-Ecuador: Ediciones Comunidec-Amazanga.

Vitery, A. (2010). Proyecto de construcción de la circunscripción territorial Kichwa de Pastaza. Nacionalidad Kichwa de Pastaza. Puyo-Ecuador. 